\title{
VII.
}

Aus dem Laboratorium der psychiatrischen und Nervenklinik der Königl. Charité (Prof. Jolly).

\section{Zur Darstellung der Axencylinderfibrillen in den markhaltigen Fasern des Centralnervensystems nebst Bemerkungen zur Histologie des Axen- cylinders im Allgemeinen.}

\author{
Von \\ Dr. Warncke, \\ Volontärarzt đer Klinik. \\ (Hierzu Tafel VI.)
}

In einer sehr ausführlichen Arbeit haben Mönkeberg und Bethe ${ }^{1}$ ) unter Vervollkommnung eines zuerst von Kupfer und Boveri angegebenen Verfahrens neue Untersuchungen angestellt, über die "Fibrillen", sowobl im normalen markhaltigen Nerven als auch im erkrankten. Wir verdanken diesen Untersuchungen die genauesten Abbildungen, die wir von Fibrillen im Axencylinder überhaupt besitzen, Abbildungen, die den Gedanken nahe legen, dass es möglich sein muss, vermittelst der Methode noch weiteren Einblick in die feinsten Verhältnisse des Axencylinders zu gewinnen, als es bisher möglich war.

Eine der vielen naheliegenden Fragen war, wie es mit dem Verhalten der Fibrillen innerhalb der centralen Bezirke markhaltiger Fasern stehe.

Die Untersuchmngen von Mönkeberg und Bethe wurden aus-

1) Die Degeneration der markhaltigen Nervenfasern der Wirbelthiere unter hauptsächlicher Berücksichtigung der Primitivfibrillen von J. Mönkeberg und A. Bethe. - Archiv für mikroskopische Anatomie und Entw.-Geschichte. 1899. 2. IV. 
schiesslich an den zunächst am meisten sich eignenden grosskalibrigen Fasern der peripheren Nervenstämme ausgeführt, während die Fasern des Centralnervensystems keine Berücksichtigung fanden, da nach den eigenen Mittheilungen der Autoren die Darstellung der Fibrillen an diesen letzteren nicht gelungen war.

Wie weit überhaupt von früberen Autoren über fibrillären Bau der centralen Markfasern etwas festgestellt ist, kann ich nicht sagen.

Soweit meine Kenntniss der fast unübersehbaren Literatur geht, dürfte das meiste, was bez. der Fibrillen gesagt ist, auf Beobachtungen an dem gewöhnlich benutzten Ischiadicus beruhen.

Nur bei Kölliker finde ich eine Notiz, nach der er das Rückenmark des Ochsen gerade zum Studium der Fibrillenbilder empfiehlt, und zwar vermittelst der Silber- und Osmium-Behandlung; der betr. Passus ist jedoch nicht ganz klar und eindeutig; Abbildungen sind nicht dabei. Vor allem ist nicht zu erfahren, ob es sich um Schnittoder Zupfpräparate handelt, und doch würden nur erstere das haben zeigen können, worum es sich bei unsern Untersuchungen handelt.

Jedenfalls ist man mit Bewusstsein und planmässig dieser Frage nicht näher getreten and das mit gutem Grund; denn alle vor BetheApaty von Fibrillen gegebenen Bilder waren nicht so deutlich, als dass man sich dadurch zu weitergehenden Untersuchungen hätte veranlasst sehen können.

Es ist also ganz erklärlich, wenn wir über Fibrillen im centralen Nervencylinder so gut wie nichts wissen.

Selbstverständlich würde es für die principielle Auffassung eines durchgehends fibrillären Baues des gesammten Nervensystems nicht als ein Hinderniss gelten können, wenn eine solche Darstellung der Fibrillen, wie wir sie Bethe für die peripheren Nerven verdanken, für andere Bezirke des Nervensystems nicht möglich wäre.

Ist doch das tinctorielle und chemische Verhalten der Axencylinder in allen sonstigen Beziehungen das gleiche, und ist ausserdem doch für die Ganglienzellen die Darstellung der Primitivfibrillen in grosser Vollkommenheit möglich.

Trotzdem schien es mir der Mühe werth, den Versuch der Anwendung des Bethe'schen Verfahrens auch auf die centralen Nervenfasern erneut in Angriff zu nehmen.

War es doch zunächst schon von Interesse, zu sehen, aus welchen Gründen die im Princip relativ einfache Methode der beiden Autoren (Fixiren dünner Nervenstämme in Osmium, Einbetten in Paraffin, Anlegen feinster Schnitte u. s. w.) gerade bei centralen Fasern nicht gelungen war. In der Arbeit selber fanden sich darïber keine Angaben. 
Dann aber schien es auch nicht ausgeschlossen, dass, wemn auch die bisherigen Untersuchungsmethoden keine Unterschiede im Bau des peripherischen und centralen Axencylinders ergeben hatten, doch bei Zuhülfenahme feinerer Methoden das Verhalten der Fibrillen Differenzen erkennen liess.

Weiterhin musste die Möglichkeit, feinste pathologische Veränderungen an den Fasern des Centralnervensystems zu finden, erst dann erreichbar erscheinen, wenn es überhaupt einen besseren Weg als bisher zur Sichtbarmachung der feinsten histologischen Elemente an denselben gab.

Endlich aber liessen sich doch vielleicht auch aus unvollkommenen Resultaten Schlüsse irgend welcher Art ziehen.

In der That möchte ich von vorne herein bemerken, dass es mir nicht gelungen ist, meine Aufgabe in so vollkommener Weise zu lösen, wie es Bethe z. B. für dte periphereu Nerven gelungen ist. Ich habe weder an peripheren, noch an centralen Axencylindern annähernd so ideale Fibrillenbilder erhalten, wie ich sie in der Bethe'schen Arbeit abgebildet finde. Ich glaube aber, dass auch meine relativ unvollkommenen Resultate um deswillen ein gewisses' Interesse verdienen, weil die Erörterung einiger technischer Fragen für weitere Arbeiten in dieser Richtung von Werth sein kann, sei es auch nur in der Weise, dass dadurch Wiederholung naheliegender aber nutzloser Versuche vermieden wird.

Sodann ist die Zahl der Arbeiten, welche sich mit der mühsamen Fibrillendarstellung beschäftigen, vorläufig noch so gering und ebenso die Zahl der Abbildungen, über dic wir verfügen, dass dadurch jede Arbeit, enthielte sie auch nur Nachprüfungen und Controlle des bisher Behaupteten, von Werth wird; namentlich im Hinblick darauf, dass diejenigen, die erstmalig Neues gefunden haben, leicht über das Ziel hinausschiessen.

Es dürfte zweckmässig sein, das von Mönkeberg-Bethe befolgte Verfahren an dieser Stelle noch einmal kurz anzugeben.

1. Dünne Nervenstämme, nicht über $1 \mathrm{~mm}$ dick, werden in natürlicher Spannung auf Kork befestigt und in 0,25 proc. Ueberosmiumsäurelösung gebracht auf 24 Stunden;

2. Wasser $4-6$ Stunden;

3. 90 pCt. Alkohol 10 Stunden oder mehr;

4. Wasser 4 Stunden;

5. Für 6-12 Stunden in neue 2 proc. Lösung von Natriumbisulphit, welchem auf je $10 \mathrm{ccm}$ direct vor dem Einlegen $2-4$ Tropfen conc, Salzsäure zugesetzt sind; 
6. Wasser $1-2$ Stunden;

7. Alkohol;

8. Xylol;

9. Paraffin.

Schnitte so dünn wie möglich, $2-3 \mu$. Aufkleben mittelst Eiweiss und Wasser. Wieder zurück in

11. Xylol;

12. Alkohol;

13. Wasser.

Bethe selber giebt nun zwei Methoden an, von denen ich die erste, von $B$ ethe als directe bezeichnete, übergehen möchte, weil sie der anderen an Zweckmässigkeit resp. Güte der Resultate, meiner Erfahrung nach, nachsteht.

Die zweite, indirecte Methode ist folgende:

14. Für 5-10 Minuten in eine auf $20-30^{\circ} \mathrm{C}$. erwärmte 1 bis 4 proc. Lösung von Ammoniummolybdat gebracht, dann 5-6 mal kurz abgespült mit destillirtem Wasser.

Es darf mit Toluidinblau dann keinen Niederschlag geben.

Der Objectträger wird an den Rändern trocken gewischt und die Schnitte

15. mit $0,05-0,1$ proc. Toluidinblau überschichtet, 5 Min. bei 50 bis $60^{\circ} \mathrm{im}$ Brütofen.

Abspülen, Alkohol, Xylol, Balsam.

Worin bestehen nun die Schwierigkeiten, die sich der Anwendung dieser Methode auf die centralen Nervenfasern entgegenstellen?

Ein einfaches mechanisches Hinderniss besteht natürlich speciell für die Fasern des Gehirns in ihrem geringen Durchmesser. Es ist nicht möglich, die Schnitte so dünn zu machen, dass von diesen feinsten Fasern gleichzeitig auf 2 Seiten der Markmantel entfernt werden könnte.

Anders liegt die Sache bei den grosskalibrigen Fasern des Rückenmarks. Aber hier kommen andere Momente erschwerend in Betracht. Der geschlängelte Verlauf der einzelnen Faser, ferner der ungleichmässige Durchmesser derselben erschweren es, dass man so wie beim peripheren Nerven auf längere Strecken die Axencylinder zweiseitig vom Mark entblössen kann.

Ferner kann man diesen Missstand nicht wie beim peripheren Nerven durch Streckung der Fasern beseitigen.

Weiterhin erschwert die Weichheit des Gewebes die Entnahme so dünner Stücke, wie sie erforderlich sind, damit die Osmiumsäure genügend schnell eindringt. 
An die Möglichkeit, ganz dünne Stücke mittelst Doppelmessers zu entnehmen, habe ich leider zu spät gedacht.

Ein Hauptgrund endlich, weshalb auch, abgesehen von allen anderen Schwierigkeiten, die Darstellung der Fibrillen im centralen Axencylinder so schwer gelingt, wird erst weiter unten gewürdigt werden können, nämlich die feinere Structur des Axencylinderplasmas resp. seine grössere Empfindlichkeit gegenüber solchen Processen, die zur Gerinnung führen oder m. a. W. das schnellere Absterben des centralen Axencylinders.

Nicht das einzelne, wohl aber die Combination dieser Factoren macht die Anwendung der Bethe'schen Methode auf die gewöhnlich gebrauchten Objecte, auf das Rückenmark von Ochsen, Kaninchen, Frosch u. dgl. schwierig. Ich sage nicht anmöglich; bei etwas Geduld dürfte es wohl gelingen, auch an diesen Objecten Fibrillen darzustellen; es würde dazu nöthig sein, an dem frisch getödteten Thier ganz schnell das Rückenmark freizulegen, mittelst feiner Scheere oder Doppelmessers eine feines Streifchen Gewebe (Längsschnitt) zu entnehmen und nach vorsichtiger Fixation auf Kork mittelst Igelstacheln nach Bethe weiter zu behandeln. Wie gesagt, ich halte es für sehr wohl möglich, nach langem Suchen in den Randpartien eines solchen Gewebsstücks fibrillenhaltige Axencylinder zu finden. Aber ich kann nach meinen Erfahrungen nicht rathen, bierauf neue Mühe zu verwenden: Die Resultate werden die Mühe in keiner Weise lohnen, weil wir einen Weg zur Feststellung wertbvoller Resultate in den spärlichen Befunden nicht vor uns haben.

Anders liegen die Verhältnisse bei eimem Object, auf welches man zunächst vielleicht nicht verfällt, welches aber als einziges Material zum Studium der centralen Fibrillen sich mir ganz gat bewährt hat, nämlich das Rückenmark kIeinerer Fische.

Das Rückenmark dieser letzteren (ich habe speciell den Sumpfkarpfen, Exemplare von $6-8 \mathrm{~cm}$ Länge benutzt) ist so dünn, dass, selbst wenn es in toto in Osmium gelegt wird, doch wenigstens in seinem grösseren Theil von dieser geniigend schnell durchdrungen wird, um gröbere Gerinnungen und damit eine Verwischung feinster Structurverhältnisse zu verbindern.

Man braucht also nicht zu quetschen oder sonstwie zu lädiren, wie man es schwer vermeiden kann, wenn man dem Rückenmark grösserer Thiere dümnere Partien entnehmen will.

Während nun aber z. B. bei entsprechend kleinen Warmblütern mit entsprechend dünnem Rückenmark das Faserkaliber ein so kleines 
ist, dass Schnitte von $2-3 \mu$ nicht genügen, um die Axencylinder auf 2 Seiten rom Mark zu entb]össen, besitzt bekanntlich ein grosser Theil der Fasern in den Vordersträngen des Fisch-Rückenmarks ein solches Kaliber, dass dạs besprochene Hinderniss hier wegfällt.

Um nun gerade diese grossen Fasern im Längsschnitt zu bekommen, mussten Segmente des Rückenmarks als Serien geschnitten werden, ein in diesem Fall verhältnissmässig einfaches Verfahren.

Das Vorgehen gestaltet sich also wie folgt:

Ein kleinerer Fisch wird schnell getödtet, das Rückenmark gleichfalls schnell freigelegt und Stücke von $2-3 \mathrm{~mm}$ Länge in verdünnte $1 / 4$ proc. Osmiumlösung gelegt. Weiterbebandlung wie oben angegeben.

Erst beim Schneiden tritt ein Unterschied ein, insofern als man sich nicht damit begnügen darf, wie beim peripheren Nerven etwa beliebig feine Partikelchen abzuhobeln, sondern es ist nöthig, wie eben bemerkt, möglichst das ganze Segment in feinste Sehnitte zu zerlegen. Man läuft anders Gefabr, gerade die relativ eng umgrenzten Gebiete der grossen Fasern zu verfehlen.

Zum Glück schneidet sich das in Osmium gehärtete Rückenmark sehr viel besser als peripherer Nerv. Schnittbänder von $2-3 \mu$ sind leicht zu erreichen.

Will man nicht das ganze Segment in Schnitte zerlegen, sondern etwa nur die Vorderstränge, so kann man sich in der Weise helfen, dass man vor der Herausnahme des Rückenmarkes aus dem Wirbelcanal die Dorsalstränge mit einer gebogenen Scheere abträgt.

Die Osmiumsäure hat demnach leichteres Spiel beim Eindringen. Andererseits ist doch bei der Zartheit des Gewebes eine Deformirung durch diesen Eingriff leicht möglicb, so dass ganz parallele Schnitte (zur Rückenmarksaxe) nicht so gut gelingen.

Ausserdem kann man von dem in toto eingebetteten Rückenmarksstück, um zu sehen, auf welcher Stelle die Ventralstränge liegen, einen Querschnitt anlegen, an dem man sich orientirt.

Natürlich muss dann der Paraffinblock entsprechend geschnitten werden.

Geht man in dieser Weise vor, so erhält man unter günstigen Umständen (Schnittrichtung genau parallel zur Rückenmarksaxe) bei einer Vergrösserung von etwa 700 zunächst Uebersichtsbilder, wie sie Abbildung I zeigt. Man hat hier annähernd dieselben Bilder der auf längere Strecken hin zweiseitig rom Ylark entblössten Axencylinder, wie man sie hat bei Schnitten etwa durch den Ischiadicus des Frosches.

Im einzelnen sieht man Folgendes: 
Wir haben zunächst eine Anzahl Markfasern, an denen der Axencylinder fast ungefärbt erscheint; die genauere Beobachtung zeigt, dass hier der Axencylinder den ganzen Markraum ausfüllt, nicht geschrumpft ist. Es sind dies Fasern, bei denen offenbar das eingetreten ist, was Bethe für den frisch osmirten Nerven behauptet hat, dass sein Plasma in eine Modification übergeführt ist, die späterhin Gerinnung und Schrumpfung verbindert. Die betreffenden Axencylinder habell doch nicht nur die Behandlung mit Alkohol und Xylol, sondern sogar die Hitze des Paraffinofens überstanden, ohne zu schrumpfen.

In diesen Fasern wird man Fibrillen erwarten, und thatsächlich findet man sie auch und zwar nur hier, in diesen Axencylindern, die sieh durch ablehnendes Verhalten gegen Farbe und Mangel der Schrumpfung charakterisiren. Diese Fasern liegen in den Randpartien des Schnittes.

Weiterhin sehen wir nun eine ganze Anzahl Fasern, die zwar nicht geschrumpft sind, wo also wahrscheinlich auch noch eine Einwirkung der Osmiumsäure wirksam war, die aber sich intensiver gefärbt haben als die anderen. An diesen ist von fibrillärer Structur nichts zu erkennen.

Eine dritte Kategorie von Fasern zeigt das uns allen geläufige Bild des compacten, zu einem Strang coagulirten, von der Markscheide retrahirten Axencylinders, während eine Reibe von Fasern deutlich alle möglichen Uebergangsstadien zwischen geschrumpften und nicht geschrumpften Axencylindern zeigen. Ueberall da, wo der Axencylinder den Markraum nicht ganz ausfüllt, haben wir intensive Färbung desselben mit den verschiedensten Farbstoffen.

Wo es den Anschein hat, als ob in ihnen noch irgend etwas von Structur erkenntlich wäre, lässt sich bei genauerer Betrachtung nachweisen, dass man es mit ganz groben Gerinnungen zu thun hat. Es ergiebt sich das schon aus der Beobachtung, dass schon an den nicht geschrumpften Fasern die fibrilläre Structur vielfach nicht kenntlich ist, sondern durch feinkörnige Gerinnung ersetzt resp. verdeckt ist. Wieviel weniger ist an den auf $1 / 6$ ihres Volumens zusammengeschrumpften Axencylindern der Nachweis einer Structur zu erwarten.

Der Umstand, dass die Fasern der letzten Kategorie im Centrum liegen, erklärt sich natürlich einfach daraus, dass selbst in diesem so dünnen Gewebsstïck die Osmiumsäure nicht schnell genug eingedrungen ist. Auf die Folgerungen werden wir weiter unten zu sprechen kommen.

Wendet man nun ganz starke Vergrösserungen an, so erhält man 
Bilder, wie sie Fig. 2, 3 und 4 zeigt, Bilder, deren Deutung nach dem bisher Gesagten keine Schwierigkeiten machen dürfte.

Bez. Il a bemerke ich, dass es sich um eine im Längsschnitt getroffene Mauthner'sche Riesenfaser mit geschrumpftem Axencylinder handelt.

Fig. 3 zeigt Querschnittsbilder bei einer Vergrösserung von ca. 700 darunter ebenfalls den einer Riesenfaser; letzteres nur zur Illustrirung der Grössenverhältnisse.

Diese Querschnittsbilder zeigen nicht eigentlich deutlich Fibrillen. Wohl hat man bei gewissen Einstellungen den Eindruck feiner Pünktchen, aber wirkliche Beweiskraft möchte ich für die Bilder in dieser Hinsicht nicht beanspruchen. Wohl aber ist trotzdem schon nach Quersehnittsbildern bestimmt zu sagen, ob eine Nervenfaser fibrillenartig ist oder nicht, sofern man im letzteren Falle den Axencylinder schon bei oberflächlichem Hinsehen fast ungefärbt weiss sieht. Er sieht so aus, wie der Axencylinder am osmirten, nicht gefärbten Nervenquerschnitt immer aussieht.

Während wir aber für gewöhnlich die geschrumpften coagulirten Axencylinder eben wegen schlechter Färbbarkeit des Osmiummaterials nicht sichtbar machen können, treten beim Bethe'schen Verfahren. (übrigens auch ohne dasselbe bei intensiver Färbung) die letateren sehr deutlich hervor, während die nicht coagulirten Axencylinder weiss bleiben.

Ich möchte an dieser Stelle darauf aufmerksam machen, dass eine Vereinfachung des Bethe'schen Verfahrens dann Platz greifen kamn, wenn man nicht deutliche Fibrillenbilder sehen will, sondern wenn man sich begnügt festzustellen, ob überhaupt und wieweit in einem Gewebsstück die Axencylinder geschrumpft sind oder nicht. Es genügt dann und giebt sehr schöne Uebersichtsbilder, wenn man einfach 5--10 Min. nach v. Gieson bei $50^{0}$ im Brütofen färbt und dann kurz abspült. Allerdings müsssen die Schnitte sehr dünn sein $(2 \mu)$.

Es dürfte hier anch der Punkt sein, sich zn fragen, ob das, was man auf Längsschnitten als Fibrillen sielıt, wirklich präformirte histologische Gebilde sind oder nicht. - Znx Zeit ist das ein Axiom, und ich möchte selbstverständlich nicht anf Grund meiner Präparate etwas gegen die Beweiskraft der Bethe'schen Abbildungen sagen. Aber da mir keine Originalpräparate zugänglich waren, kann ich nicht umhin, nach den Fibrillenbildern zu urtheilen, die jch selbst habe anfertigen können. Obwohl dieselben namentlich an den peripheren Nerven sehr gut gelungen warell, musste doch stets ein gewisser Zweifel bleiben, ob diese zarten Fäden nicht doch vielleicht nur Gerinnungsprodukte seien. Und gar 
die Fibrillen an den centralen Fasern documentiren sich als solche eigentlich nur durch den Parallelismus mit den erheblich deutlicheren der peripheren Nerven.

Ich glaube indessen, dass man, auch ohne in dieser Frage definitiv Stellung zu nehmen, ruhig vom Fibrillenbild der Axencylinder sprechen kann, indem dasselbe auf jeden Fall den Werth eines Aequivalentbildes im Sinne $\mathrm{N}$ issl's hat. Und aus diesem Grunde glaube ich, auch wenn die von mir gegebenen Abbildungen centraler Axencylinderfibrillen nicht von idealer Deutlichkeit sind, doch auf einiges aufmerksam machen zu dürfen, was sich mir als Resultat der gewonnenen Bilder aufdrängte.

Zuwächst erweckt es den Anschein, als ob die Fibrillen in den centralen Fasern feiner wären als diejenigen, die man im peripheren Nerven sieht. Doch möchte ich diese Behauptung nur mit einer gewissen Reserve aussprechen.

(Bezüglich anderer Fragen, z. B. ob Theilungen vorkommen, ob Varicositäten sich finden, $o b$ die Fibrillen aus hintereinander gelagerten Körnern bestehen, möchte ich kein bestimmtes Urtheil abgeben.)

Dagegen möchte ich auf einen deutlichen wesentlichen Unterschied zwischen centralen und peripheren Axencylindern hinweisen.

Es ist Mönkeberg und Bethe nicht entgangen, dass die Darstellung der Fibrillen nur an dünnen Nervenstämmen gelingt resp. in den Randpartien dickerer, wo also die Osmiumsäure relativ schnell eingewirkt hat. Dass nun die in der Tiefe gelegenen Fasern das Fibrillenbild nicht mehr zeigen, kann zwei Gründe haben: entweder die Fasern haben an dem Zeitpunkt, wo die Osmiumsäure zu ihnen gelangt, ihre vitale chemische Constitution in Folge Absterbens nicht mehr besessen, oder aber die Einwirkung der Osmiumsäure in der Peripherie hat activ in der Tiefe chemische Processe zur Folge gehabt, welche die Faser verändert haben. Beides ist denkbar.

In jedem Falle aber ist das Erhaltensein des Fibrillenbildes ein Beweis, dass die vitale Constitution noch erhalten war in dem Moment, wo die Osmiumsäure mit der Faser in Berührung kam, and wir haben so unter Umständen in dieser Einwirkung der Osmiumsäure einen gewissen Massstab, ob und wie lange die vitale Constitution einer Faser erbalten bleibt.

Mönkeberg und Bethe haben diesen doch sehr naheliegenden Schluss meines Wissens nicht gezogen.

Wenden wir diese Sätze auf unsere Abbildungen an, so lässt sich sagen, das Plasma der centralen Nervenfasern muss ausserordentlich 
schnell seine vitale Constitution verlieren, da nur in einem Theil der Randfasern eines ganz dümnen Gewebsstückes die Axencylinder nicht geschrumpft sind.

Es wird das recht klar, wenn man daneben die Thatsache halt, dass in dem fast ebenso dicken lschiadicus eines grossen Frosches noch 24 Stunden nach dem Tode jede Faser fibrillären Bau zeigt nnd $z$ war in unveränderter Dentlichkeit.

Ausserdem ist bei vielen, noch nicht geschrumpften Rückenmarksfasem der fibrilläre Bau durch eine feine gleichmässige Gerinnung verdeckt resp. ersetzt, während bei peripheren Fasern diese Structur stets erst dann schwindet, sobald eine deutliche Schrumpfung eintritt.

Zusammengefasst scheint sich mir zu ergeben, dass zunächst beim Kaltblüter das Plasma der peripheren Nervenfaser sehr viel länger und energischer als das der ceutralen seinen vitalen Chemismus post mortem behält.

Das Verhalten resp. diese Deutung scheint mir um deswillen beachtenswerth, als es dem entspricht, was wir auch sonst über das Erhaltensein der Erregbarkeit nach dem Tode bei centralen und peripheren Fasern wissen. Demn wenn auch der genaue Nachweis der Dauer der postmortalen Erregbarkeit centraler Fasern wegen der Dazwischenschaltung der Ganglienzellen kaum möglich ist, ist jedenfalls bis jetzt der Nachweis einer postmortalen Erregbarkeit der Rückenmarksfasern nur für kurze Zeit erbracht, während bekannt ist, dass der vor Austrocknen bewahrte Frosch-lschiadicus noch Tage lang nach dem Tode seine Erregbarkeit behält.

Noch in einer zweiten Hinsicht scheinen unsere Abbildıngen lehrreich, nämlich dadurch, dass sie direct nebeneinander den "normalen" Axencylinder, sodann den arteficiell geschrumpften und endlich eine Reihe sehr charakteristischer Uebergangsformen bringen. Es wird bei diesem Anblick vor Allem ohne Weiteres deutlich, wie aussichtslos es ist, bei den sonst üblichen Härtungsmethoden etwa noch feinere Structurdetails am Axencylinder finden zu wollen.

Man würde derartige Versuche vielleicht gar nicht gemacht haben, wenn man nicht unter dem ständigen Einfluss der Bilder der in Chromsäure und Alkobol gehärteten Axencylinder vergessen hätte, in wie hohem Maasse wir es dabei mit Kunstproducten zu thun haben. Der frisch osmirte, nicht geschrumpfte Nerv zeigt einen ca. 5-6 fach grösseren Umfang des Axencylinders als man ihn bei allen übrigen Fixirungsmethoden zu Gesicht bekommt. 
Was nun die erwähnten Zwischenformen betrifft, so könnte man zunächst glauben, dass man es mit Fasern zu thun hat, die in bestimmten Momenten des Schrumpfungsprocesses fixirt sind. Die Bilder erinnern durchaus an diejenigen, die man bei eintrocknenden plasmatischen Gebilden sieht.

Indessen zeigt genauere Ueberlegung, dass wir es hier mit späterer Schrumpfung partiell hereits fixirter Fasern zu thun haben müssen.

Wir dürfen also diese Bilder für die Theorie des Schrumpfungsprocesses nur mit Vorsicht verwerthen.

Ich möchte die Erörterung dieser anscheinend unwichtigen Frage hier aus dem Grunde auschliessen, weil sie uns auf etwas principiell Wichtiges führt, nämlich auf das Verbältniss von Fibrillen und Perifibrillärsubstanz.

Nach Mönkeberg und Bethe würde, wenn anders ich die betreffenden Ausführungen recht verstanden habe, die Schrumpfung des Axencylinders sich so vollziehen, dass die Fibrillen zu einem Strang zusammenschnurren und die Perifibrillärsubstanz an die Aussenseite dieses Stranges tritt.

Verhielte sich das wirklich so, so wäre es natürlich ein weiteres Glied in der Kette von Beweisen dafür, dass beide Bestandtheile des Axencylinders nicht eigentlich organisch miteinander verbunden sind, sondern mehr räumlich neben- und ineinander existiren und sich im Grunde wie Fremdkörper gegeneinander verhalten.

Bekanntlich ist die Frage nach der Beschaffenheit der Fibrillenzwischensubstanz bisher nicht irgendwie sicher entschieden. Sie drängte sich für den Histologen auf, sobald man nach Kölliker's Vorgang den Axencylinder als aus feinsten Fibrillen bestehend ansah, die durch eine Kittsubstanz miteinander verbunden sein sollten.

Aber noch in der neuesten Auflage seiner Gewebelehre kann der Verfasser derselben nichts weiter thun, als die verschiedenen Hypothesen nebeneinander stellen, denen zu Folge die betreffende Substanz entweder fest oder festweich oder flüssig sein soll.

Entschieden ist diese Eintheilung keine glückliche.

Begriffe wie "fest" oder "festweich" in ihrer Anwendung auf ein jedenfalls sehr wasserreiches Gebilde können kaum geeignet sein, Klarheit zu schaffen.

Es dürfte auch thatsächlich weniger auf die Consistenz der Fibrillenzwischensubstanz ankommen, als auf die Frage: Sind beide Bestandtheile des Axencylinders organiseh oder mechanisch miteinander verbunden, haben wir es zu thun mit Protoplasmafäden, die mechanisch 
in einer mehr weniger flüssigen Kittmasse liegen, oder sind sie organisch untrennbar mit derselben verbunden?

Est wenn man sich für die erstere Auffassung entschieden hat, muss man sich weiter fragen: Ist das die Fibrillen Trennende eine Flüssigkeit oder etwas, was man als Kittmasse, Kittsubstanz bezeichnen kann, was dam irgendwie den Charakter eines besonderen Gewebebestandtheils tragen müsste?

Mönkeberg und Bethe äussern sich über diesen Punkt nicht, aber aus den Abbildungen und dem Namen Perifibrillärsubstanz schliesse ich, dass sie an eine Art von Nervenserum (Kupfer) nicht denken, sondern eher an eine Art von weichem Nervenkitt.

Ein Coaguliren der im Nervenserum flottirenden Fibrilleu zu einem Strang mit Verdrängung des Wassers nach aussen wäre nun allenfalls vorstellbar. Indessen dürfte eine solche Auffassung; so sehr sie durch manche Bilder gestützt wird, kaum der Wirklichkeit entsprechen. Thatsächlich ist aber das auch nicht die Meinung jener Autoren.

Wie man sich aber ein Zusammenschnurren der Fibrillen, wenn dieselben in eine Art von "Substanz" eingebettet sind, vorstellen soll, ist mir unverständlich.

Trotzdem will ich nicht so weit gehen, diesen Modus des Schrumpfens für alle Fälle als unmöglich hinzustellen.

Bestreiten muss ich aber entschieden, dass, soweit centrale Fasern in Betracht kommen, irgend etwas Derartiges nachweisbar wäre.

Stets fülltentweder der meist fibrillenhaltigeAxencylinder denganzen vom Markumsehlossenen Hohlraum aus, dabei eine charakteristische Reactionslosigkeit gegen Farben zeigend, oder er fült diesen Hohlraum uicht aus: dann ist er intensiv gefärbt, der Zwischenraum stets ungefärbt, und enthält nie spuren irgendwie gefärbter, als "Substanz" sich charakterisirender Gewebsbestandtheile, die man als herausgetretene Perifibrillärsubstanz auffassen könnte. Stets ist in diesem Falle auch die fibrilläre Structur verschwunden, und stets bildet der geschrumpfte Axencylinder eine homogene Masse.

Es scheint mir demnach ausgeschlossen, den Vorgang anders aufzufassen, als bisher wohl meist geschehen, nämlich so, dass die aus dem Axencylinder austretenden Bestandtheile aus Wasser resp. seröser Flüssigkeit bestehen, welches aber nicht mechanisch zwischen den Fibrillen herausgedrängt ist, sondern infolge eines chemischen Processes aus seinen Verbindungen frei geworden ist. Dafür spricht in erster Linie 
der Umstand, dass ein tinctoriell ganz anderes Verhalten Platz greift, sobald einmal der Axencylinder angefangen hat zu schrumpfen.

Ich glaube, dass aus unsern Bildern wenigstens das deutlich hervorgeht, dass der Schrumpfungsvorgang nicht darin besteht, dass die Fibrillen sich einfach mechanisch dichter aneinanderlegen.

Formen wie die sub II a und IIe wären schlechterdings unmöglich. Allenfalls könnte man sich fragen, ob im geschrumpften Axencylinder, wie wir ihn sich ja überall deutlich präsentiren sehen, nicht doch eine innere Schicht, die Fibrillen, und eine äussere, die an den Rand gedrängte Zwischensubstanz, besteht. Aber ich meine, man müsste das, sei es auf Quer-, sei es anf Längsschnitten irgendwo sehen.

Ich muss deshalb bis auf weiteres daran festhalten, dass die von Bethe behauptete Art des Schrumpfungsprocesses, die eben doch von principieller Bedeutung für die Frage des Axencylinderbaues wäre, wenigstens für die centralen Fasern, sich zunächst nicht bestätigt.

Ich habe es deshalb vorgezogen, von Axencylinderplasma und fibrillärer Structur desselben zu sprechen, im Sinne einer organischen Zusammengehörigkeit beider Bestandtheile des Axencylinders.

Voraussetzung der ganzen Deduction ist natürlich - was nach meinen Präparaten nicht ganz deutlich ist —, dass die "Axencylinderfibrillen" nicht doch etwa Gerinnungsproducte feinster Art sind, in welchem Falle der ganze Streit gegenstandslos wäre.

Zum Schluss möchte ich auf einen mehr nebensächlichen Vortheil aufmerksam machen, der bei Serienschnitten durch die Vorderstränge des Fisch-Rückenmarks sich ergiebt und der darin besteht, dass ein für das Studium der Nervenfaser besonders geeignetes Object einer genaueren Besichtigung zugänglich wird, nämlich die Mauthner'schen Fasern.

Zwar kann man auch auf Querschnitten des Rückenmarks die Verhältnisse speciell des Axencylinders studiren; indessen wird das Innere desselben doch nicht in solcher Weise zugänglich wie vermittelst ganz dünner Längsschnitte.

Die Grösse der Fasern gestattet es dann, mit grosser Sicherheit Einblick in Verhältnisse zu gewinnen, über die sonst in Folge der Kleinheit der Objecte Zweifel bestehen können.

Ohne eine erschöpfende Behandlung der zahllosen strittigen Fragen zu versuchen, die bez. der markhaltigen Faser bestehen, möchte ich nur bemerken, dass von einer Reihe anderweitig beschriebener Elemente ich nichts habe sehen können, nämlich von der sog. Axencylinderscheide, von Gefässen im Nerven, von Kittsubstanztrichtern, die nach 
Caplan Axencylinder und Markscheide organisch verbinden sollen, endlich nicht von eigentlicher Structur des Markmantels.

Leider war in den von mir untersuchten. Fällen der Axencylinder bereits geschrumpft, so dass ich speciell die Verbältnisse der Fibrillen an einem so grossen Objecte nicht studiren konnte. Ich möchte es aber für der Mühe werth halten, den Versuch einer Darstellung der Fibrillen gerade an den Mauthner'schen Fasern zum Gegenstand einer besonderen Arbeit zu machen.

Das Wesentliche der vorstehenden Ausführungen möchte ich wie folgt kurz zusammenfassen:

1. Die Darstellung von Axencylinderfibrillen nach dem BetheNönkeberg'schen Verfahren ist nicht nur an den peripheren, sondern auch an den centralen Faserı möglich bei Benutzung geeigneten Materials.

2. Als solches empfiehlt sich das Rückenmark kleinerer Fische.

3. Das Fibrillenbild des osmirten Nerven hat, einerlei, ob es sich um Gerinnungsproducte oder präformirte Structur handelt, die Bedeutung eines Aequivalentbildes für eine bestimmte vitale Constitution des Nerven.

4. Die fibrilläre Structur ist an peripheren Nerven erheblich länger post mortem und deutlicher nachweisbar als an den centralen Fasern.

5. Wahrscheinlich haben wir darin den anatomischen Ausdruck für das Bestehen teinster chemischer und physiologischer Unterschiede zwischen peripheren und centralen Fasern, speciell für das leichtere Absterben der centralen Fasern.

6. Die Darstellung von Bethe-Mönkeberg, wonach die Schrumpfung des Axencylinders sich unter Austritt der Perifibrillärsubstanz vollzieht, während die Fibrillen sich aneinanderlegen, die hierin zum Ausdruck kommende mechanische Gegenüberstellung von Fibrillen und Perifibrillärsubstanz findet bez. der centralen Fasern zunächst keine Bestätigung.

Zum Schluss ist es mir eine angenehme Pflicht, Herrn Prof. Köppen für die Anregung zu der Arbeit sowie für die Controlle derselben meinen wärmsten Dank auszusprechen.

\section{Erklärung der Abbildungen (Taf. VI).}

I. Längsschnitt duroh die Vorderstränge des Fjsch-Rückenmarks (mittlere Vergrösserung), Behandlung nach Mönkeberg-Bethe. a) Axencylinder an 
der Peripherie gelegen, nicht geschrumpft, fibrillenhaltig. b) Völlig geschrumpfter Axencylinder. e) Uebergangsform. d) Axencylinder nicht geschrumpft, homogen granulirt, dunkler tingirt als a.

II. Einzelne Fasern im Längsschnitt bei stärkster Vergrösserung. a) Riesenfaser, Axencylinder geschrumpft. b) Axencylinder, der nebeneinander Fibrillen und homogenes Aussehen zeigt. c) Axencylinder, dessen Schrumpfung nur partiell durch die Osmiumsäure verhindert. d) Gewöhnliches Bild des zu einem sōliden Strang zusammengeschrumpften Axencylinders. e) Uebergang der Fibrillen in den geschrumpften Axencylinder.

III. Fibrillenhaltige Nervenfaser aus dem Ischiadicus des Frosches. Ranvier'sche Einschnürung.

IV. Querschnittsbilder vom Axencylinder aus dem Fisch-Rückenmark. a) nicht geschrumpft. b), d) Uebergangsformen. e) Riesenfaser. e), f) Maximal geschrumpft. 


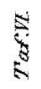

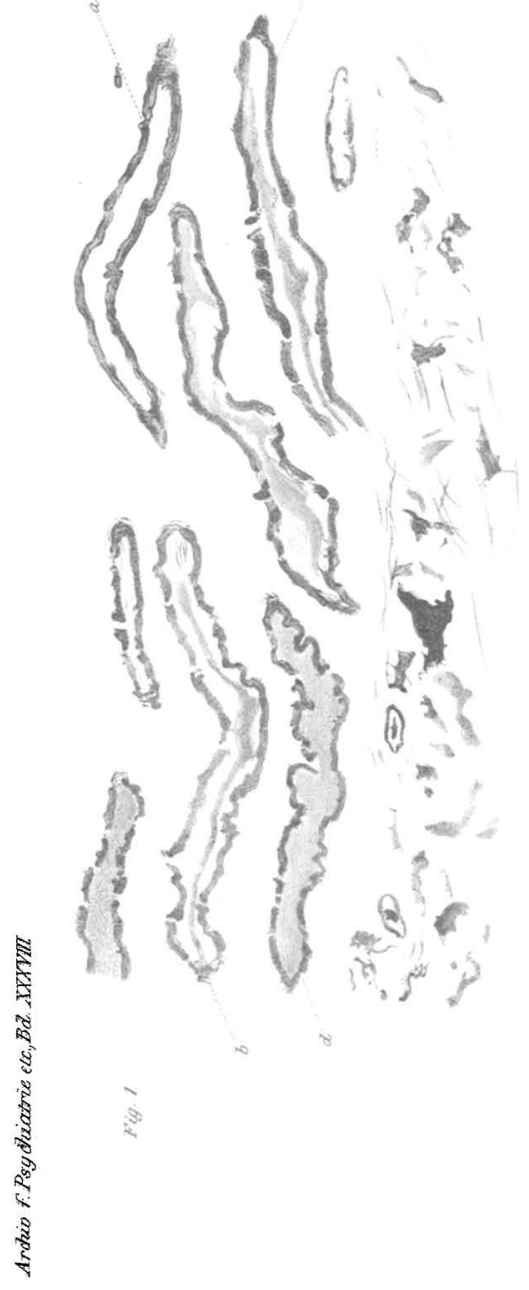

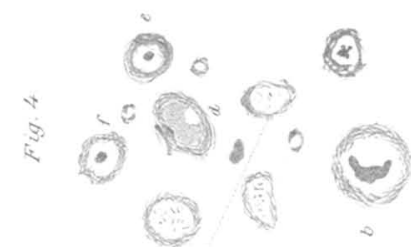
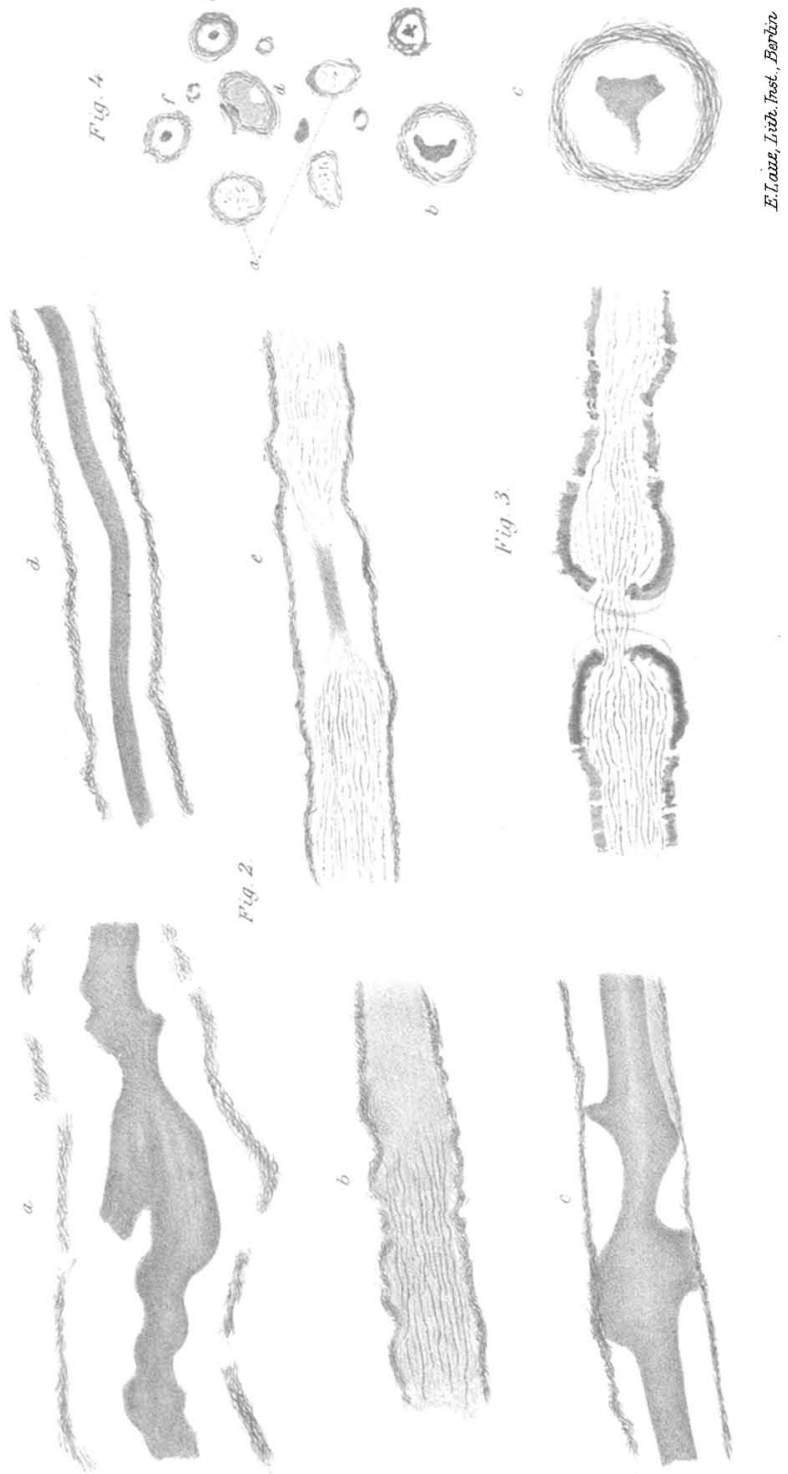